\title{
La conception aristotélicienne de la servilité dans l'Éthique et la Politique
}

\author{
LOUISE RODRIGUE Cégep de Saint-Jérôme
}

RÉSUMÉ : Novateur à plusieurs égards, l'ouvrage influent de Richard Bodéüs, Le philosophe et la cité, paru en 1982, propose notamment une réinterprétation de la notion complexe de phrónēsis. C'est dans cette foulée que se situe la présente contribution, dont l'objectif est d'analyser une de ses formes plus modeste, l'économie, dans sa partie qui implique le rapport avec les personnes non-libres. À la lumière des observations de Richard Bodéüs sur le commandement, les positions aristotéliciennes concernant la relation maître-esclave seront remises en perspective. Cette démarche conduira, en deuxième partie, à montrer l'extension de la notion servilité, plus large qu'on ne l'admet généralement, ce qui donnera à voir en quoi la nature humaine est esclave, selon le mot du Stagirite (Métaphysique A 2, 982b29-30).

ABSTRACT: Ground breaking in many ways, Richard Bodéüs' influential book, Le philosophe et la cité, published in 1982 (The Political Dimensions of Aristotle's Ethics, 1993), offers a reinterpretation of the complex notion of phrónessis. In the same line of inquiry, this article analyzes one of the more modest forms of phrónesis, namely economy, more specifically the part of economy that involves the relationship with unfree people. The Aristotelian views on the master-slave relationship are put into perspective in light of Bodéüs' observations on command. This approach leads to pointing out the full meaning of the concept of servility, which is deeper than is generally recognized, allowing readers to grasp how "in many respects human nature is servile," according to the Metaphysics (A, 2, 982b29-30).

Mots-clés: Aristote, Richard Bodéüs, servilité, éthique, politique

La philosophie du Stagirite, comme toutes les autres, a ses têtes d'affiche, au nombre desquelles on compte le sophós, le spoudaīos, le phrónimos, le pepaideuménos. Celle, moins évidente, du nomothète fait aussi partie du panorama des études aristotéliciennes depuis la parution en 1982 de l'ouvrage influent de

Dialogue 59 (2020), 51-68

(C) Canadian Philosophical Association/Association canadienne de philosophie 2020 doi:10.1017/S0012217320000153 
Richard Bodéüs, Le philosophe et la cité. Recherches sur les rapports entre morale et politique dans la pensée d'Aristote. Novatrice à de nombreux égards, notamment dans sa manière de considérer le corpus aristotélicien qui ne tient plus de l'approche «génétiste», cette étude a définitivement établi la nature politique du projet aristotélicien touchant la philosophie humaine. La thèse d'après laquelle l'Éthique et la Politique s'adressent au législateur ${ }^{1}$ et visent sa formation fait désormais autorité ${ }^{2}$. Les implications d'une telle interprétation sont multiples, et c'est ainsi que Richard Bodéüs explique, à propos de la vertu du politique, la phrónēsis, qu'elle se décline en plusieurs formes, dont la principale, qu'il qualifie de nomothétique, se trouve au cœur des préoccupations d'Aristote dans ses discours de l'Éthique et de la Politique. En plus de démontrer l'importance de la figure du politique dans l'œuvre du Stagirite, Bodéüs reprend à nouveaux frais l'exégèse de la notion complexe de phrónēsis et en détaille le caractère polymorphique. Se détachant de la conception traditionnelle qui envisage la phrónēsis comme une excellence régissant principalement les actes individuels, l'analyse que propose Bodéüs de l'Éthique à Nicomaque (EN VI, 8, 1141b23 sq.) distingue trois formes que peut prendre cette vertu. Quand elle est politique au sens large (A.), il s'agit de la nomothétique (qui est architectonique) (1.), et au sens étroit (2.), elle est délibérative (a) ou judicative (b). L'économie constitue la deuxième forme de cette vertu ${ }^{3}$ (B.), et la troisième (C.) se donne comme phrónēsis au sens spécifique, relative à l'individu. Son analyse est synthétisée dans le tableau suivant :

phrónēsis [au sens générique]

A. [politikè au sens large]

1. nomothetike- -arkhitektonike

2. politike [au sens étroit]
a) bouleutike
b) dikastike

B. oikonomía

C. phrónēsis [au sens spécifique] - hē perì hautòn kaì héna ${ }^{4}$

1 R. Bodéüs (1982, p. 18, et plus spécialement chapitre II, §III.1, p. 118-125). L'auteur signale que la qualité de nomothète doit s'entendre dans une acception fort large (ibid., p. 129).

2 Par exemple, Pierre Pellegrin reprend cette thèse pour expliquer au lecteur la démarche d'Aristote dans l'introduction des Politiques parus chez Flammarion (1990, p. 29), et fait de même dans l'«Introduction à la philosophie pratique» parue dans l'édition de 2014 des Euvres complètes (Aristote, 2014b, p. 1972).

3 Comme les politiques, les personnes qui savent tenir leur maison, signale Aristote, sont des gens sagaces, car ils sont capables de voir ce qui est bon pour eux-mêmes et ce qui l'est pour les êtres humains (EN VI, 5, 1140b8-10).

4 R. Bodéüs (1982, p. 126). Nous avons translittéré les termes grecs. Le tableau apparaît, modifié, dans R. Bodéüs (2004, p. 67). 
C'est à la deuxième forme, plus modeste, que la présente contribution entend s'intéresser. Tirer les conséquences d'un tel classement impliquant l'économie domestique peut être saisissant, surtout considérant la relation de type despotique, laquelle est constitutive de l'économie (avec les relations maritale et parentale; Politique I, 3, 1253b1-14). Le tableau élaboré par Bodéüs offre en effet la possibilité de recadrer la relation du maitre et de l'esclave dans une perspective plus large qui, si elle n'est pas celle de la phrónēsis proprement dite, n'en entretient pas moins certains liens avec cet état, que nous tâcherons ici d'identifier. Cette démarche contribuera au débat actuel entourant les positions aristotéliciennes à propos de la servilité, dans la mesure où la discussion demeure plutôt centrée sur l'esclave et, de ce fait, néglige volontiers l'apport critique du Stagirite sur cette réalité de son époque; nous tâcherons de souligner cet apport en fournissant une description de la relation despotique du point de vue du maître. Cet angle, peu exploité, conduira par ailleurs à montrer l'extension de la notion de servilité, plus large qu'on ne l'admet généralement, et à constater que l'asservissement peut représenter une menace, même pour les personnes qui semblent le plus à l'abri de cette condition. L'économie, telle qu'identifiée par Bodéüs parmi les formes de phrónēsis, s'en trouvera donc clarifiée - du moins dans son aspect despotique - de même que sera éclaircie l'attitude du Stagirite face à cette dernière, dont le caractère subversif a été mis en évidence par notre auteur dans la foulée de ses travaux sur la philosophie politique d'Aristote.

\section{La relation despotique au sein de l'économie}

Il est certain que dans l'absolu, les deuxième et troisième formes de la phrónēsis mentionnées ci-devant n'ont pas la même portée que la nomothétique; pourtant, l'autorité de l'économe sur les actions concernant la vie domestique (de même que celle de l'individu sur ses actes) est l'équivalent de l'autorité que détient le politique dans la cité, et ce, d'autant plus que concrètement parlant, tout individu vit dans une famille, laquelle fait partie d'une cité ${ }^{5}$. Si la nomothétique laisse loin derrière elle l'économie et la phrónēsis au sens spécifique, selon Bodéüs, c'est principalement en raison du caractère intellectuel de son œuvre $^{6}$, qui va de pair avec sa dimension architectonique. C'est peut-être aussi attribuable à la quantité et à la nature des personnes concernées par son exercice. En effet, la phrónēsis au sens spécifique se rapporte aux actes individuels qui ne concernent que l'agent lui-même; l'économie règle les actions impliquées dans le fonctionnement du ménage, lequel comprend époux, enfants et esclaves, la politique culminant dans l'activité législative qui s'applique à l'ensemble des citoyens. Ce niveau d'impersonnalité croissant fait de la

\footnotetext{
5 R. Bodéüs (1982, p. 127).

6 R. Bodéüs (1982, p. 127-128).
} 
sphère domestique un moyen terme entre l'individu et la cité, et à l'intérieur même de la domesticité, la relation despotique semble ménager une transition vers une activité impliquant des personnes encore plus éloignées du sujet. Ainsi, à côté des actes concernant le seul agent se trouvent ceux qui font intervenir des membres de la famille : les enfants, au premier chef, puisqu'Aristote les définit comme d'autres soi-même à l'état séparé (EN VIII, 14, 1161b27-28), mais aussi l'époux dans la mesure où l'être humain est fait pour le couple ${ }^{7}$, dans le cadre duquel les conjoints se complètent. Dans cette perspective, les relations parentale et maritale constitutives de l'économie se rapprochent du rapport à soi-même; or c'est un peu moins vrai de la relation despotique. À tout le moins, Aristote n'a pas cru bon de décrire les esclaves dans de pareils termes, préférant les catégoriser à l'aide de la notion d'avoir. Il écrit à cet égard que l'esclave par nature ne s'appartient pas à lui-même, mais appartient à un autre (Pol. I, 4, 1254a14-15), formule indiquant que l'esclave, tout en étant humain, est la possession (ktêma) de son maître. Le philosophe précise qu'une possession est un instrument servant à l'action (órganon praktikón; Pol. I, 4, 1254a17); en tant qu'instrument, l'esclave se définit d'abord comme un $\operatorname{corps}^{8}$, de sorte que sa fonction principale est de rendre service avec son corps (Pol. I, 13, 1259b25) — il est attaché aux travaux serviles du corps, les tâches les plus serviles étant celles qui font le plus appel à l'activité physique (Pol. I, 11, 1258b38). Les individus ayant pour tâche de se servir de leur corps (hè tồ sōmatos khrêsis), dit Aristote, et dont c'est le meilleur parti qu'on puisse tirer d'eux, ceux-là sont esclaves par nature ${ }^{9}$ (Pol. I, 5, 1254b18-19). Cette description très succincte indique qu'à la différence des membres de la famille, qui sont des autres soi-même, l'esclave appartient au maître et en constitue une partie, à savoir une partie animée et séparée de son corps (Pol. I, 6, 1255b12; voir encore EE VII, 9, 1241b18-24). Ces deux caractéristiques de la servilité semblent faire de la relation despotique une manière, à l'intérieur du cadre domestique, d'étendre la portée de l'autorité du sujet en position de commandement, passant de ce qui est considéré comme lui-même à ce qui lui appartient ou lui est assigné, comme une étape intermédiaire vers une forme plus complexe ou achevée d'autorité.

L'interprétation que propose Bodéüs de ce qu'Aristote entend par phrónēsis signifie encore davantage en ce qui concerne l'économie. Considérant que la vertu présente un contraire, il se trouve que la phrónēsis en admet au moins

7 EN VIII, 14, 1162a17. La même idée est exprimée dans l'Éthique à Eudème (EE VII, $10,1242 \mathrm{a} 23)$. À la rigueur, l'amitié pourrait s'ajouter ici, même si elle n'est pas de nature domestique, car Aristote envisage l'ami en tant qu'autre soi-même (EE VII, 12, 1245a30).

8 R. Weil (1982, p. 342), parle de «l'homme-corps».

9 L'excellence des esclaves se trouve dans leur corps, souligne E. Garver (1994, p. 188). 
un. Le fait est qu'Aristote en identifie deux, du moins si l'on prête foi au tableau des états moyens et extrêmes apparaissant en Éthique à Eudème, où la phrónēsis est opposée à la fourberie et à la naïveté ( $E E$ II, 3, 1221a12). Peut-être est-ce dans cet esprit que le propos de Bodéüs est essentiellement tourné vers ce qu'Aristote appelle le «vrai politique» ${ }^{10}$ par opposition au «faux politique», qu'il n'a de cesse de dénoncer ${ }^{11}$. Le Stagirite explique en effet que la plupart des politiques ne méritent pas leur nom, car ils ne sont pas vraiment des politiques; est tel l'individu qui décide les belles actions pour elles-mêmes, tandis que la plupart des gens embrassent cette vie pour l'argent et le profit indu ( $E E$ I, 5, 1216a22-25). Dans la mesure où l'économie compte à titre de forme possible de phrónēsis, alors il est vraisemblable qu'elle s'oppose à (au moins) un vice apparenté à ce qu'Aristote décrit dans l'Éthique à Eudème - s'il existe de faux politiques, il est permis de supposer qu'il y a de faux économes. Il va de soi que le vrai (et le bon) économe s'acquitte correctement de ses fonctions d'époux, de père et, en principe, de maître. Dans ce cas, il exerce une autorité parentale de type royal, une autorité maritale de type aristocratique et une autorité despotique de type tyrannique, selon le très fameux passage de l'Éthique à Nicomaque (EN VIII, 12 1160a24-35). En revanche, dans l'éventualité où il ne mérite pas son nom, le chef domestique se comporte comme un tyran avec ses enfants et transforme la communauté conjugale en oligarchie. Il est clair d'après cet extrait qu'existe un revers ou une déviation de l'économie dans ses parties parentale et maritale; cela est moins clair en ce qui concerne la partie despotique. Mais Aristote semble l'admettre ailleurs : en Politique I, 6, il signale que le gouvernement exercé par un maitre peut ne pas l'être convenablement, ce qui cause du désavantage des deux côtés (1255b8-9). Ce cas de figure, anonyme, ne se détermine pas tout à fait au moyen du même critère que les deux autres : les déviations dans l'ordre parental et conjugal surviennent quand la personne qui commande le fait dans son propre intérêt, au mépris de celui des gens qu'elle gouverne - comme les dirigeants des régimes politiques déviés, qui sont de faux politiques (voir $E N$ VIII, 12, $1160 \mathrm{a} 30$ et sq.). Cependant, on ne saurait reprocher à l'économe de poursuivre d'une certaine manière son propre intérêt et son enrichissement. C'est l'essence même de l'association d'un maître avec ses esclaves, qui est de nature tyrannique, puisqu'elle exécute l'intérêt du maître ( $E N$ VIII, 12, 1160b29-30; voir aussi Pol. III, 6, 1278b32-35). Il y a pourtant une limite à la recherche de l'intérêt personnel, au-delà de laquelle on quitte l'économie pour la chrématistique. Cette dernière, explique Aristote, a pour but la richesse et la possession des valeurs, alors que l'économie se sert de la richesse en vue d'autre chose, nommément, la vie heureuse. C'est précisément la borne fixée à l'accroissement des richesses, qui empêche de verser dans l'excès (Pol. I, 9, $1257 \mathrm{~b} 18$ et sq.). L'observation

10 R. Bodéüs (1982, p. 123, où la note 18 renvoie à $E N$ I, 13, 1102a7-13).

11 Sur cette distinction, voir R. Bodéüs (2004, p. 5-30). 
indique, pour le despotisme, que sa limite est marquée par le but de la domesticité, à savoir la vie heureuse : dans cette perspective, le maître ne devrait exercer son commandement sur l'esclave que dans la mesure où cela sert le bien de la maisonnée. Le faire en outrepassant cette borne cause du dommage aux deux parties impliquées - à l'esclave, il va sans dire, et par le fait même au maître, qui a tout intérêt à ne pas endommager sa propriété ${ }^{12}$. Ainsi, l'économe qui remplit adéquatement son rôle de maître a conscience qu'un esclave n'est pas seulement un instrument, mais un instrument animé, c'est-à-dire doté d'une sorte d'âme qui le rend humain; or, en tant qu'être humain, soutient Aristote, l'esclave peut susciter de l'amitié (EN VIII, 13, 1161a34-b8). C'est possiblement ce qui contribue à expliquer l'inclusion de l'économie parmi les dispositions constitutives de la phrónēsis.

L'interprétation donnée par Bodéüs de la phrónēsis indique qu'elle est d'ordre «épitactique» ${ }^{13}$ ou prescriptive (epitaktiké; EN VI, 11, 1143a8. Il ne fait pas de doute qu'il s'agit là d'une caractéristique positive lorsqu'il est question de la plus haute forme de phrónēsis, et que c'est là en partie ce qui définit sa nature architectonique - la nomothétique n'est commandée par aucune autre science ${ }^{14}$ — et justifie sa place en tête du classement des différentes variétés de cette vertu. Or, dans le cas de l'économie, il semble que la dimension épitactique puisse à l'occasion prendre un tour défavorable, du moins lorsque le rapport despotique est en cause. Le fait est que ce type de rapport, même convenable, n'est pas très satisfaisant - ce n'est pas en tant que maître qu'une personne s'accomplit. Aristote explique à cet effet que la science du maître (epistēmè despotikē, et ce n'est pas elle qui le fait maître) est la science de l'utilisation des esclaves (hēe khrēsikê doúlōn), et consiste à savoir leur ordonner (epitáttein) ce qu'ils doivent faire. Cette science, dit-il, n'a rien de grand ni de vénérable, de sorte que les maîtres qui ont le moyen d'éviter le tracas de son exercice en laissent le soin à un intendant, tandis qu'eux-mêmes s'occupent de politique ou de philosophie (Pol. I, 7, 1255b30-38). Il n'y a rien de noble, ajoute le Stagirite, à se servir (khrēsthai) d'un esclave en tant qu'esclave, et donner des ordres (epitaxis) concernant les tâches indispensables ne fait en rien partie de ce qui est beau; c'est pourquoi la vie du maître d'esclave ne saurait être la meilleure ${ }^{15}$ (Pol. VII, 3, 1325a25-27). Ainsi, l'exercice de l'autorité despotique comporte un aspect indésirable, à telle enseigne qu'Aristote recommande de le déléguer. Dans le cas où il n'est pas possible de confier à d'autres la tâche de commander les parties non libres de la maisonnée, le philosophe signale que le maître, dans la mesure où il voit

12 Comme le signale E. Lévy (1989, p. 207).

13 R. Bodéüs (1982, p. 70); voir encore R. Bodéüs (1990, p. 109).

14 R. Bodéüs (1982, p. 126).

15 Ce n'est pas en donnant des ordres aux esclaves que le maître s'accomplit (voir E. Garver, 1994, p. 189). 
l'humain dans l'esclave ${ }^{16}$, peut commander autrement qu'en donnant des ordres (epitáxei), c'est-à-dire au moyen d'avertissements ${ }^{17}$ (Pol. I, 13, 1260b5-7). Un tel exercice du despotisme, au sein duquel l'aspect épitactique a été si l'on peut dire adouci, à défaut de faire le bonheur du maître, rend la tâche moins pénible pour lui. Ce faisant, le maître met en outre l'esclave sur la voie de l'affranchissement, lequel semble d'ailleurs constituer l'aboutissement souhaitable de la servitude dans la perspective aristotélicienne (Pol. VII, 10, $\left.1330 \mathrm{a} 32-33^{18}\right)$. Pareille relation maître-esclave contribue à anoblir le commandement : si ce dernier reste au niveau du nécessaire, il n'en demeure pas moins que les avertissements, puisqu'ils consistent à donner les raisons du commandement $^{19}$, humanisent l'esclave en reconnaissant en lui la présence d'une certaine rationalité ${ }^{20}$. Être sensible à l'admonestation (et aux encouragements), en effet, montre que le sujet obéit à la raison et donc y participe d'une certaine façon (EN I, 13, 1102b28-1103a1). Voilà donc qui concourt à appuyer, si besoin est, la place modeste de l'économie dans le tableau des formes de phrónēsis : l'aspect épitactique qu'elle implique exige d'être atténué, sous peine d'avilissement pour la personne qui l'exerce.

Ainsi l'exercice convenable du despotisme suppose-t-il un commandement ne se fondant pas seulement sur les ordres; cela semble être l'une des conditions auxquelles cette partie de l'économie pourrait traduire une attitude qui tiendrait

16 Selon V. Goldschmidt (1984, p. 64), tout l'effort d'Aristote consiste à voir dans l'esclave un être humain; c'est peut-être pourquoi sa position sur l'esclavage a pu faire l'effet d'une attaque contre cette institution (ibid., p. 74).

17 W. W. Fortenbaugh (2015, p. 403) signale qu'en cela, Aristote se distingue de Platon; l'auteur renvoie en outre à la Rhétorique (Rhét. II, 3, 1380b18-20), où le philosophe explique que l'esclave s'indigne moins d'être puni si le châtiment est précédé d'une réprimande.

18 R. Bodéüs (2004, p. 93). Le passage est aussi commenté par V. Goldschmidt (1984, p. 76) et par P. Pellegrin (1982, p. 354). Il s'agit précisément de l'attitude adoptée par Aristote lui-même à l'égard de ses esclaves : il avait prescrit, dans son testament, d'affranchir sa maisonnée servile (à propos de ce passage, voir Aristote, Euvres, 2014a, p. 1400, note 20). Voir aussi J. Brunschwig (1979, p. 31), E. Lévy (1989, p. 209), P. Garnsey (1996, p. 127) et N. D. Smith (1991, p. 144), lequel ajoute cependant que les Grecs distinguaient entre les esclaves affranchis et les personnes libres (ibid., p. 48, note 27) : l'affranchissement ne conférait pas à l'individu le statut de citoyen non plus que les privilèges se rattachant à ce statut.

19 R. Bodéüs (2004, p. 94); c'est aussi l'avis de M. Deslauriers (2003, p. 218).

20 R. Bodéüs (2003, p. 348). Voir encore W. W. Fortenbaugh (1977, p. 137). Selon J. Karbowski (2012, p. 346), la fonction propre de l'être humain, l'activité rationnelle, admet plusieurs modalités; la délibération et la méditation, certes, mais aussi la délibération «domestique» et le fait d'être à l'écoute de la raison. 
de la vertu. Il est possible de clarifier cette manière d'opérer considérant que les impératifs du maître peuvent se traduire en termes de causalité finale. Aristote établit à ce propos un parallèle éclairant : la médecine et la santé sont toutes deux dites commander, mais la première donne des ordres en vue de la seconde. Il en va de même, poursuit Aristote, pour le principe méditatif : il est ce en vue de quoi la sagacité ${ }^{1}$ donne des ordres (hou héneka hē phrónēsis epitáttei; EE VIII, 3, 1249b14-15; héneka epitáttei; EN VI, 13, 1145a9). L'analogie entre la santé et le principe méditatif en tant que causes finales s'applique aussi à la divinité qui, explique le philosophe, ne commande pas en donnant des ordres (ou gàr epitaktikôs árkhōn ho théos; EE VIII, 3, 1249b13-14). On ne saurait affirmer plus clairement que l'aspect épitactique du commandement, même droit, ne convient pas à ce qu'il y a de meilleur. Cela dit, la manière dont la cause finale exerce le commandement (sans donner d'ordre) pourrait trouver écho dans le cadre de la relation despotique. Le même passage de l'Éthique à Eudème donne une indication à cet égard (1249b16-23) : ce qui favorise la méditation, note Aristote, c'est pour l'âme d'avoir le moins possible conscience de l'autre partie (de la partie irrationnelle) en tant que telle ${ }^{22}$. La remarque pourrait suggérer, dans le cadre du despotisme, que le commandant ne peut diminuer le recours aux ordres que dans la mesure où le commandé se comporte de moins en moins en tant que tel. Le premier a ainsi le moins conscience possible de l'existence du second - le maître ne peut relâcher son emprise sur l'esclave que dans la mesure où celui-ci se trouve dans un état tel qu'il requiert le moins de commandes possible.

Ces considérations indiquent une particularité du rapport de commandement, signalée par Bodéüs lorsqu'il constate la relative inutilité de la législation pour les assujettis qui sont «parvenus à saisir les principes vrais qu'elle exprime en termes généraux» ${ }^{23}$. Si la loi est moins nécessaire pour qui en comprend la portée, alors il pourrait vraisemblablement en aller de même pour les ordres donnés aux esclaves : ils deviennent moins utiles pour ceux qui en connaissent

21 Il semble s'agir ici de la forme la plus achevée de la phrónēsis (nomothétique), en tête du tableau mentionné ci-dessus. Son aspect épitactique n'a pas la teneur qu'il présente au niveau économique (despotique), dans la mesure où il s'exerce sur des êtres libres et ne concerne pas le domaine de l'indispensable.

«Donc, l'option qui, dans le choix et la possession des biens naturels, favoriserait par-dessus tout la méditation du dieu, qu'on y emploie le corps, les richesses, les amis ou les autres biens, devrait être la meilleure option et la plus belle limite à fixer, alors qu'une option qui, soit par manque de biens, soit par excès, empêche de servir le dieu et de méditer, est, elle, une option mauvaise. Or cela tient à l'âme, et la meilleure limite que l'âme puisse se fixer est d'avoir le moins possible conscience de sa partie irrationnelle en tant que telle» (Aristote, Euvres I, 2014a, p. 374).

23 R. Bodéüs (1982, p. 131). 
les raisons. C'est ainsi que le rapport despotique exercé convenablement est voué à s'amenuiser, et peut-être même à disparaître; le maître, explique Bodéüs, perd de sa nécessité pour l'esclave qui a corrigé sa nature ${ }^{24}$. Conscient de ce fait, le (véritable) économe sait que la domination n'est pas une fin en soi, et qu'il est nécessaire qu'elle reste ordonnée à l'objectif qu'est la vie heureuse (Pol. VII, 2, 1325a1-15); à ce titre, elle ne constitue qu'un moyen, et doit donc demeurer à l'intérieur de limites strictes. L'idée qu'un commandement convenable se restreint au maximum apparaît dans un passage du Mouvement des animaux. Aristote, mentionnant le cas d'une cité dont les lois sont bien faites, explique qu'il n'est pas besoin d'un monarque qui devrait être présent chaque fois qu'il se passe quelque chose, puisque chacun accomplit lui-même ce qui lui revient, comme cela a été ordonné ( $M A, 10,703 \mathrm{a} 29-34)$. Dans cette perspective, il appert qu'un pouvoir exercé convenablement fait peu appel à la coercition et réduit son aspect épitactique. En regard du despotisme, cette remarque tend à signifier que plus les ordres du maître sont clairs (en prenant éventuellement la forme d'avertissements parce qu'il en donne les raisons), moins il est contraint d'intervenir auprès des assujettis pour que les choses se fassent. Sous ce rapport, commander bien consiste d'une certaine manière à commander moins. Une telle observation préside peut-être même au classement des différentes formes de phrónēsis, la plus éminente impliquant très peu d'intervention de la part du commandant lui-même.

\section{D'autres états réputés serviles}

Si la nécessité des lois, comme le montre Bodéüs, ne fait aucun doute par rapport à la formation du citoyen et à sa poursuite du bonheur ${ }^{25}$, elle apparaît en revanche moins clairement pour la personne qui a conquis l'autonomie parfaite, c'est-à-dire qui détient la phrónēsis sous toutes ses formes (personnelle, économique et politique). En effet, parallèlement à la possibilité pour l'esclave de dépasser sa condition se trouve celle pour le citoyen de s'émanciper des lois. Or, ainsi que l'observe Bodéüs, la route est longue depuis la servilité jusqu'à l'affranchissement des 10 is $^{26}$, en raison notamment des multiples obstacles qui se dressent le long de cette route. En fait, il importe de reconnaître qu'échapper à l'esclavage ou au rapport despotique ne garantit nullement la liberté - ce n'est pas parce qu'un individu est maître qu'il est libre pour autant. La servilité est effectivement un péril qui guette tout un chacun, dans la mesure où la nature humaine est esclave (Métaphysique A, 2, 982-b29-30). Par ce constat inquiétant, Aristote donne à penser qu'à côté de l'esclavage proprement dit existent des états qui lui ressemblent, lesquels sont susceptibles d'affecter des personnes libres, et même celles dont la situation est enviable. Autrement dit, la

\footnotetext{
24 R. Bodéüs (2004, p. 95, note 66).

25 R. Bodéüs (1982, p. 108-114).

26 R. Bodéüs (2004, p. 96).
} 
condition des individus libres, que le philosophe appelle eleutheroi ou pepaideuménoi $^{27}$, recèle des écueils qui risquent de faire basculer les sujets dans un état qui rappelle la condition opposée, les empêchant ainsi de réaliser l'autonomie parfaite. Aristote indique discrètement ces difficultés, qui vont maintenant être soulignées comme autant de mises en garde pour ceux qui pensent n'être pas concernés par la servilité.

Si la liberté exclut les occupations assujetties à l'indispensable, alors celles qu'exécutent les artisans sont aussi touchées. Aristote compte les tâches accomplies par les travailleurs manuels qui, comme leur nom l'indique, vivent du travail de leurs mains, au nombre de celles qui sont serviles, comme les travaux domestiques (sauf dans le cas où la personne les exécute pour elle-même; Pol. III, 4, 1277a35-b7). Il signale même en Rhétorique qu'il est beau de n'exercer aucun métier manuel dans la mesure où le propre d'une personne libre est de ne pas aliéner son existence à un autre (Rhét. I, 9, 1367a30-31). Ainsi apparaît la dimension servile du travail manuel : d'une part, il amène l'ouvrier à servir avec son corps, et ce faisant peut affliger le corps (Pol. VIII, 3, 1337b5-15); d'autre part, il fait en sorte que l'ouvrier ne s'appartienne pas, considérant qu'il est au service d'un maître d'œuvre dont il exécute le projet. C'est donc sans surprise que les métiers besogneux, ainsi que ceux qui sont centrés sur la rentrée d'argent, adoptés pour pourvoir au nécessaire, semblent constituer un obstacle à la belle existence (EE I, 4, 1215a20-32). À cela, il faut ajouter l'aspect spécialisé de telles occupations, qui rend les «vils artisans» utiles à la communauté politique dans un seul domaine (Pol. VIII, 4, 1338b34-35 ${ }^{28}$ ). Le fait que la plupart des ouvriers soient des gens riches (Pol. III, 5, 1278a25) ne change rien à l'affaire; au contraire, cette particularité peut les éloigner encore davantage du beau en les orientant vers les richesses matérielles.

Or, ce n'est pas tout d'être un individu disposant d'un loisir suffisant, étant donné qu'il est encore possible de se préoccuper exagérément de son corps, attitude qui tient en quelque sorte de la servilité, et constitue de ce fait un obstacle à la conquête de l'autonomie. Aristote signale à ce propos qu'il n'est utile ni à la santé ni aux activités civiques d'avoir une constitution d'athlète. Certes, il ne faut pas non plus être trop délicat; cependant, la constitution entraînée à l'effort doit tendre aux activités propres à la personne libre, et non aux efforts violents ne visant qu'un seul but comme c'est le cas pour l'athlète (Pol. VII, 16, 1335b5-11). Étant donné qu'elle apporte santé et force (Pol. VIII, 3, 1338a20), le Stagirite encourage le recours à la gymnastique dans l'éducation des jeunes; cependant, la gymnastique n'est indiquée qu'après la puberté, dans la mesure où les exercices ne font pas obstacle à la croissance ni ne

27 R. Bodéüs (1982, p. 203).

28 Ce passage est relevé par P. Pellegrin (2017, p. 142). L'auteur précise que cette classe de riches indignes de la liberté menace la cité en constituant un groupe de pression politiquement dangereux. 
gâtent la vigueur (Pol. VIII, 4, 1338b38-1339a $6^{29}$ ). Il appert ainsi que la spécialisation dans les exercices physiques d'une seule sorte s'oppose à une certaine polyvalence caractéristique de la liberté; en d'autres termes, s'il importe d'avoir le goût de l'effort ou du travail, il se doit toutefois d'être dénué de servilité (Rhét. I, 5, 1361a8). Dans le cas de l'athlète, cette dernière prend moins la forme d'un service au moyen du corps que d'un souci excessif pour celui-ci, lequel souci fait en sorte que le sujet ne s'appartienne pas tout à fait, considérant que le corps est d'une certaine manière «l'autre» de ce qui définit en propre l'être humain (une chose qui pense, $E N$ IX, 4, 1166a23).

Dans la perspective aristotélicienne, les artistes se trouvent dans une situation comparable à celle des athlètes : leur spécialisation les place en état de servilité. Aristote convient qu'il est nécessaire de connaître la musique, ne serait-ce que pour être en mesure d'apprécier et de juger les belles mélodies (Pol. VIII, 6, 1340b30-40). Cependant, comme le rappelle Bodéüs, il considère les musiciens professionnels comme des artisans (Pol. VIII, 5, 1339b8-10), parce que leur virtuosité a pour but de plaire aux autres - aux auditeurs (Pol. VIII, 6, $\left.1341 \mathrm{~b} 12^{30}\right)$. Au contraire, l'apprentissage de la musique convenant à la personne de bien ne doit en rien avilir le corps ni rendre inapte aux exercices civiques (1340b6-8), et l'habilité ne doit se déployer qu'en vue de la propre vertu de l'exécutant (1341b12). Alors que les musiciens professionnels peuvent voir leur corps avili en raison des mouvements qu'ils exécutent ${ }^{31}$ et restent soumis à leur auditoire ${ }^{32}$, les personnes ayant reçu une éducation non spécialisée (non professionnelle) s'épargnent ces maux physiques et visent leur propre vertu lorsqu'il s'agit de musique. La pratique artistique peut donc verser dans la servilité en raison du dommage corporel qu'éventuellement elle cause, et parce qu'elle fait en sorte que le professionnel ne s'appartient plus tout à fait - c'est sans doute en partie pourquoi Aristote réprouve l'éducation professionnelle ${ }^{33}$.

Or, le fait est que, même libres et dégagés de toute contrainte professionnelle, il arrive aux êtres humains de choisir un mode de vie servile. C'est du moins le qualificatif qu'emploie Aristote lorsqu'il parle des individus qui mènent une

29 Ce passage est commenté par C. Lord (1982, p. 59). L'auteur signale l'attitude critique d'Aristote envers l'éducation dispensée chez les Laconiens, lesquels abrutissent les enfants avec des exercices pénibles, comme si cela incitait au courage (Pol. VIII, 4, 1338b10-15).

30 R. Bodéüs (1982, p. 196-197).

31 Voir l'allusion à la déformation du visage provoquée par le hautbois ou la flûte (Pol. VIII, 6, 1341b1-5), un passage relevé par C. Lord (1982, p. 53).

32 En encourageant le désir de plaire et de s'adapter à un auditoire, l'éducation musicale professionnelle se révèle dommageable pour l'âme, de remarquer encore C. Lord (1982, p. 53).

33 R. Bodéüs (1982, p. 197). 
existence consacrée aux plaisirs corporels. La masse des gens, observe le philosophe, préfère la vie faite de jouissances physiques; c'est le choix de la plupart de ceux pour qui la question du genre d'existence à privilégier se pose. En faisant ce choix, la masse se montre complètement servile (andrapodōdeis; EN I, 3, 1095b19-20), les plaisirs corporels étant à la portée du premier venu et même de l'être servile (andrápodon; EN X, 6, 1177a8). Seules les personnes serviles, dit le passage eudémien (andrápodon; EE I, 5, 1215b34-35), vivent en fonction des plaisirs corporels. Le statut juridique de personne libre ne suffit donc pas pour échapper à la servitude ${ }^{34}$; encore faut-il se comporter comme tel, et il semble bien que la vertu de tempérance soit requise à cette fin. La vie digne d'une personne libre, en effet, peut conduire à la mollesse en l'absence de cette excellence. C'est pourquoi Aristote définit la vie heureuse comme une vie tempérée digne d'un individu libre ${ }^{35}$.

La nature humaine peut ainsi se montrer esclave en raison du choix du genre d'existence, de la réalisation de tâches besogneuses, ou de la pratique sportive et artistique. La servilité menace toutefois de surgir même au niveau du genre de vie le meilleur dans la perspective aristotélicienne; le plus pernicieux des asservissements, en effet, est de nature intellectuelle et guette même les personnes ayant bénéficié d'une éducation libérale. Il en est question au dernier livre de la Politique, sur lequel Bodéüs attire l'attention lorsque, traitant de l'auditeur des discours politiques ${ }^{36}$, il signale les mérites d'une telle éducation (contre l'éducation professionnelle). Il observe, entre autres, que selon Aristote, il faut enseigner les choses indispensables, mais parmi elles seulement celles qui ne feront pas des individus qui les pratiquent de simples artisans, c'est-à-dire des personnes dont le corps, l'âme ou l'intelligence est inapte aux activités vertueuses (Pol. VIII, 1, 1337b4-10). Les connaissances utiles doivent être enseignées, d'après le philosophe, non seulement pour leur utilité, mais aussi parce qu'il est possible par leur intermédiaire d'acquérir beaucoup d'autres connaissances. Tel est le cas de l'étude des lettres (pour les affaires, l'administration domestique et nombres d'activités civiques), et même du dessin : on enseigne ce dernier moins pour éviter aux gens des erreurs dans leurs achats

34 Dans cette perspective, remarque R. Bodéüs (2003, p. 350), le jugement d'Aristote sur l'esclave naturel s'applique aussi à ceux de ses contemporains (et des nôtres) qui sont de condition juridiquement libre et n'ont pour seul but dans l'existence que la satisfaction des plaisirs corporels.

«Socrate dit qu'elle doit être assez grande pour qu'on puisse mener une 'vie tempérée', comme si cette expression voulait dire une 'vie heureuse'. Voilà qui est trop général, sans compter qu'on peut mener une vie à la fois tempérée et misérable. Une meilleure définition serait : une vie tempérée et digne d'un homme libre [...]» (Pol. II, 6, 1265a30-35; voir Aristote, Euvres complètes, 2014b, p. 2355).

R. Bodéüs (1982, p. 195-201). 
personnels que parce que le dessin fait voir la beauté des corps, favorisant ainsi un meilleur jugement des œuvres artistiques. Chercher partout l'utile, en effet, ne sied pas du tout aux personnes libres (Pol. VIII, 3, 1338a15-19 et 1338a36-b4). De plus, il n'est pas indigne pour de tels individus de prendre part jusqu'à un certain point aux sciences libérales. Le fait de maîtriser ces disciplines, explique Aristote, confère au sujet une certaine autonomie puisqu'il est apte à juger de ce qui est bien ou mal dit dans un discours; l'individu cultivé, signale-t-il, est capable de juger pour ainsi dire de tout (Parties des animaux I, $\left.1,639 \mathrm{a} 1-9^{37}\right)$. Pareille culture générale met à l'abri de l'esclavage intellectuel face aux experts autoproclamés dans toutes les disciplines, car le sujet est à même de discerner ce qui vaut (ou ne vaut pas) la peine d'être écouté.

Cependant, la précision d'Aristote importe : il écrit que l'étude des sciences libérales convient «jusqu'à un certain point». Bodéüs le signale expressément : l'éducation idéale, en quelque domaine que ce soit, répugne à l'excès d'instruction ${ }^{38}$. En effet, la spécialisation, dommageable aux niveaux corporel et professionnel, l'est semblablement au niveau intellectuel : le fait d'apporter à l'étude une assiduité excessive, allant jusqu'à la minutie, comporte de l'avis du philosophe certains inconvénients (Pol. VIII, 1, 1337b15-18). La nature de ces inconvénients n'étant pas expliquée, on a avancé l'hypothèse que la spécialisation produirait un type particulier de servitude intellectuelle confinant la réflexion du spécialiste à l'unique sujet qu'il connaît trop bien, rabaissant en quelque sorte l'intelligence ${ }^{39}$. À cet égard, l'expression «jusqu'à un certain point» pourrait signifier que l'étude doit être circonscrite dans les limites qui suffisent aux objectifs recherchés - pousser plus loin le souci de rigueur est un labeur excessif. Un exemple donné par Aristote éclaire l'idée de la mise en perspective de la connaissance : le médecin, pour pouvoir soigner l'œil, doit connaître le corps entier (EN I, 13, 1102a17-15). À l'inverse, l'hyperspécialisation peut nuire aux objectifs globaux de l'étude en faisant perdre l'ensemble de vue. C'est ainsi que Bodéüs remarque que la personne éduquée libéralement, le pepaideuménos, n'est pas un expert ou un savant ${ }^{40}$; celui qui s'engage sur cette voie s'expose à un type particulier d'aliénation consistant à avoir la «pensée besogneuse» (Pol. VIII, 2, 1337b14), et qui peut par exemple prendre la forme d'une obsession du détail allant jusqu'à l'insignifiance. À l'image de la nature servile inca-

37 Le passage est relevé par R. Bodéüs (1982, p. 202, note 3); C.D.C. Reeve le commente aussi (2012, p. 254-255).

38 R. Bodéüs (1982, p. 203).

39 C.D.C. Reeve (2012, p. 255).

40 R. Bodéüs (1982, p. 198); l'auteur précise que cette personne peut juger correctement de l'œuvre de l'expert. 
pable de délibérer globalement (Pol. I, 13, 1260a12 ${ }^{41}$ ), l'individu en situation de servitude intellectuelle se montre inapte à une réflexion générale ou d'ensemble.

\section{Des cas d'exception}

Ce n'est pas tout, car la servilité peut encore se manifester de cette façon en regard de l'action et de l'étude : si on se livre à ces dernières pour soi-même, pour ses amis ou par vertu, elles ne sont pas indignes d'une personne libre, mais le faire pour d'autres personnes revêt un caractère mercenaire ou servile (Pol. VIII, 2, 1337b17-22). Ainsi échappent à la servilité les gestes posés pour eux-mêmes (ceux qui perfectionnent le sujet), et les actes qui sont accomplis pour un ami (qui est un autre soi-même; $E E$ VII, 12, 1245a30), ces exceptions étant tout aussi valables sur le plan de la pensée. Or, lorsqu'Aristote envisage la possibilité de la pensée servile, renvoie-t-il par là au sujet qui mettrait sa connaissance au service d'autrui, un peu comme le musicien professionnel joue de son instrument pour le plaisir du public et en vue d'un salaire? Si tel est le cas, toutes les sciences qui rapportent seraient susceptibles de traduire la servilité. Il existe néanmoins une exception à cet égard, comparable à celles que créent l'amitié véritable et la vertu éthique; il s'agit de la seule science libre, la seule qui soit en vue d'elle-même, qui n'est recherchée pour aucun autre avantage, à la manière dont la personne libre est celle qui vit pour elle-même et non pour quelqu'un d'autre (Mét. A, 2, 982b24-28). Comme Aristote traite de la philosophie dans les lignes précédant cette description, il est permis de croire qu'elle constitue la science libre en question. C'est en tout cas ce que suggère un passage de la Politique où Aristote explique que ceux qui souhaitent trouver en eux-mêmes la cause de leur agrément peuvent se tourner vers la philosophie (car les autres plaisirs requièrent la compagnie d'autrui; Pol. II, 7, 1267a11-13). S'ajoute à cela la finale de l'Éthique à Nicomaque ${ }^{42}$, où le Stagirite insiste sur le caractère indépendant de l'activité qui définit essentiellement le bonheur : la méditation, dit-il, est autosuffisante, car le sage, livré à lui-même, peut l'exercer (EN X, 7, 1177a27-b1). Il ajoute qu'elle constitue la seule activité à laquelle on tienne pour elle-même - le sujet n'en retire rien excepté le bénéfice de méditer (b1-2). Le bonheur de l'intelligence, souligne-t-il, est indépendant ( $E N \mathrm{X}, 8$, 1178a22). L'aperçu de la sagesse donné en Métaphysique A confirme que la science qui ne se rapporte pas à l'utile ${ }^{43}$ apparait plus sage que les autres (reliées aux nécessités de la vie; Mét. A, 1, 981b17-20). Cette «supériorité du non-

41 La traduction de R. Bodéüs (2004, p, 81, note 15), qui est le premier à rendre hólōs par «globalement», éclaire de façon nouvelle la condition de l'esclave.

42 Voir Euvres (2014a, p. 1546, note 13), où les traducteurs de la Métaphysique, Annick Stevens et Christian Rutten, commentant A, 2, 982b25-27, renvoient à l'Éthique à Nicomaque, livre X, chapitres 7-8.

43 C'est le cas de la philosophie; elle n'est pas assujettie à une fin utilitaire ni monétaire, comme le rapporte Aristote avec l'anecdote sur Thalès, à qui les gens reprochaient 
utilitaire» se manifeste aussi dans l'idée que les maîtres d'œuvre ne sont pas plus sages selon leur aptitude à l'action, mais bien selon la possession de la raison des choses et selon la connaissance des causes (Mét. A, 1, 981b5-7). Tous ces passages indiquent clairement que la liberté intellectuelle exclut la visée utilitaire, même si elle demeure compatible avec un but extérieur à soi-même, par exemple dans le cas où le sujet a en vue son ami. La personne qui vit en fonction d'une autre fait preuve d'une attitude servile, sauf s'il s'agit d'un ami, précise Aristote à l'occasion du traitement de la magnanimité ( $E N$ IV , 8, 1124b31-1125a1), entendu que l'ami est un autre soi-même (EN IX, 9, 1170b7).

Ainsi, échapper définitivement à la servilité requiert une attitude de maître d'œuvre; il semble bien que ce puisse être le cas de deux façons différentes. Aristote explique à ce propos que parmi les disciplines maîtresses, la plus souveraine et la plus éminente (málista arkhitektonikês) est la politique — au sens A.1 dégagé dans le tableau présenté par Bodéüs et reproduit plus haut ${ }^{44}$. En effet, argumente-t-il, la politique dispose (khrēon) des autres savoirs utiles pour les cités; en outre, même les plus honorables des capacités lui sont subordonnées (par exemple la conduite des armées); enfin, elle use (khrōménēs) de tous les savoir-faire, prescrivant par la loi ce que l'on doit exécuter et ce dont il faut se garder, de sorte que sa propre fin contient celle de toutes les autres disciplines ${ }^{45}$. Or la politique ou la sagacité (EN VI, 8, $1141 \mathrm{~b} 23-24)$, toute architectonique qu'elle soit, n'a pas autorité sur la sagesse, pas plus que la médecine n'a autorité sur la santé, Aristote observant que la médecine n'a pas la santé à son service (ou gàr khrêtai autêi), mais au contraire voit à son avènement. Pareillement, la sagacité donne des ordres (epitáttei) en vue de la sagesse, mais ne l'a pas à ses ordres (EN VI, 13, 1145a6-9). La remarque montre que chacune des vertus intellectuelles présente une dimension architectonique, l'une parce qu'elle commande (ou détient une autorité, ou du moins use de ce qui lui est subordonné, car elle est épitactique), l'autre parce

(suite)

l'inutilité de la philosophie en raison de sa pauvreté, alors que c'est peut-être là, au contraire, le signe de sa supériorité (Pol. I, 1, 1258b6-19).

R. Bodéüs (1982, p. 126). Voir la note 4 de cet article.

45 «Or, comme on peut l'imaginer, c'est l'objectif de la discipline la plus souveraine et la plus éminemment maîtresse. Et telle est la politique, visiblement. En effet, c'est elle qui dispose quels sont les savoirs dont on a besoin dans les cités, quelle sorte de savoirs chaque groupe de citoyens doit acquérir et jusqu'à quel point. D'autre part, nous voyons que même les plus honorables des capacités lui sont subordonnées, comme la conduite des armées, l'économie, l'art oratoire... Et, dès lors qu'elle use de tous les savoir-faire qui restent, prescrivant en outre, par la loi, ce qu'on doit exécuter et ce dont il faut se garder, sa propre fin est à même de contenir celles de toutes les autres disciplines, de sorte que cette fin doit être le bien humain» (EN I, 1, 1094a27-b7; voir Aristote, 2014a). 
qu'elle est ce en vue de quoi le commandement s'exerce. Cette différence s'appuie sur l'idée que donner des ordres n'est pas la seule manière de commander et confirme que la dimension épitactique du commandement, quand elle n'est pas avilissante, gagne en tous les cas à être exercée de la manière la moins autoritaire ou interventionniste possible.

Quoiqu'il en soit, il appert que la phrónēsis, telle que redéfinie par Bodéüs dans Le philosophe et la cité, demeure une conquête de haute lutte, dans la mesure où la mise en œuvre de ses différentes formes peut rencontrer de multiples obstacles, inhérents à la nature humaine, dont le Stagirite affirme qu'elle est esclave à plusieurs égards. Cette donnée de la condition humaine, le nomothète mieux que personne en connaît la portée, et la tâche que Bodéüs décrit comme étant la sienne vise à son dépassement. C'est possiblement dans cet esprit que Bodéüs a choisi comme épigraphe à son ouvrage un mot de Diogène Laërce selon lequel Aristote lui-même, questionné sur le profit qu'il avait retiré de la philosophie, aurait répondu : «celui de faire, sans qu'on me l'ordonne (anepitáktōs), ce que font certains sous la crainte qu'inspirent les lois» ${ }^{46}$. La remarque, en quelque sorte, indique l'apport du philosophe à la cité - en plus d'instruire le nomothète, il montre la voie à suivre pour atteindre l'autonomie, que le Stagirite semble donner comme l'aboutissement heureux de l'existence humaine.

\section{Références bibliographiques}

Aristote

1903 Ethica Nicomachea, Recognovit Franciscus Susemihl, editio tertia curavit O. Apelt, Leipzig, Teubner.

Aristote

1957 Politica, Recognovit adnotatione critica instruxerunt W. D. Ross, Oxford, Clarendon.

Aristote

1991 Ethica Eudemia, recensuerunt brevique adnotatione critica instruxerunt R. R. Walzer, J. M. Mingay; praefatione auxit J. M. Mingay, Oxford, Oxford University Press.

Aristote

2014a Euvres I, éditées par Richard Bodéüs, Paris, Gallimard («Bibliothèque de la Pléiade»).

Aristote

2014b Euvres complètes, sous la direction de Pierre Pellegrin, Paris, Flammarion.

46 Dans Vies et doctrines des philosophes illustres, livre V, 20 (Diogène Laërce, 1999, p. 574). 
Bodéüs, Richard

1982 Le philosophe et la cité. Recherches sur les rapports entre morale et politique dans la pensée d'Aristote, Paris, Les Belles Lettres.

Bodéüs, Richard

1990 «Savoir politique et savoir philosophique», dans G. Patzig, éd., Aristoteles' „Politik”, Akten des XI. Symposium Aristotelicum Friedrichschafen/Bodensee, 25.8.-3.9.1987, Göttingen, Vandenhoeck \& Ruprecht, p. 102-123.

Bodéüs, Richard

2003 «Aristote. La justice et la cité», dans La philosophie d'Aristote, Paris, Presses Universitaires de France, p. 317-415.

Bodéüs, Richard

2004 Le véritable politique et ses vertus selon Aristote. Recueil d'études, Louvain/Paris, Peeters.

Bonitz, Hermann

1955 Index aristotelicus, Graz, Akademische Druck-U. Verlagsanstalt.

Brunschwig, Jacques

1979 «L'esclavage chez Aristote», Cahiers philosophiques, vol. 1, p. 20-31. Deslauriers, Marguerite

2003 «Aristotle on the Virtues of Slaves and Women», Oxford Studies in Ancient Philosophy, vol. 25, p. 213-231.

Fortenbaugh, William W.

1977 «Aristotle on Slaves and Women», dans J. Barnes, M. Schofield et R. Sorabji, dir., Articles on Aristotle, vol. II, New York (NY), St-Martin's Press, p. 135-139.

Fortenbaugh, William W.

2015 «Aristotle on Women: Politics I 13.1260a13», Ancient Philosophy, vol. 35, p. 395-404.

Garnsey, Peter

1996 Ideas of Slavery from Aristotle to Augustine, Cambridge, Cambridge University Press.

Garver, Eugene,

1994 «Aristotle on Natural Slaves: Incomplete Praxeis and Incomplete Human Beings», Journal of the History of Philosophy, vol. 32, p. 173-195.

Goldschmidt, Victor

1984 «La théorie aristotélicienne de l'esclavage et sa méthode», dans Écrits I. Études de philosophie ancienne, Paris, Vrin, p. 63-79.

Karbowski, Joseph

2012 «Slaves, Women, and Aristotle's Natural Teleology», Ancient Philosophy, vol. 32, p. 323-350.

Laërce, Diogène

1999 Vies et doctrines des philosophes illustres, Paris, Librairie générale française. 
Lévy, Edmond

1989 «La théorie aristotélicienne de l'esclavage et ses contradictions», dans M.-M. Mactoux et E. Geny, dir., Mélanges Pierre Lévêque 3, Anthropologie et société, Paris, Les Belles Lettres, p. 197-213.

Lord, Carnes

1982 Education and Culture in the Political Thought of Aristotle, Londres, Cornell University Press.

Pellegrin, Pierre

1982 «La théorie aristotélicienne de l'esclavage : tendances actuelles de l'interprétation», Revue philosophique, vol. 172, n 2, p. 345-357.

Pellegrin, Pierre

1990 Introduction aux Politiques, Paris, GF-Flammarion, p. 5-76.

Pellegrin, Pierre

2017 L'excellence menacée. Sur la philosophie politique d'Aristote, Paris, Classiques Garnier.

Reeve, Christopher D. C.

2012 Action, Contemplation, and Happiness. An Essay on Aristotle, Cambridge/Londres, Harvard University Press.

Smith, Nicholas D.

1991 «Aristotle's Theory of Natural Slavery», dans D. Keyt et F.D. Miller, dir., A Companion to Aristotle's Politics, Oxford/Cambridge, Blackwell, p. 142-155.

Weil, Raymond

1982 «Deux notes sur Aristote et l'esclavage», Revue philosophique, vol. 172, $n^{\circ} 2$, p. 339-344. 